\title{
GLOBAL ChaOS SyNCHRONIZATION OF HYPERCHAOTIC NEWTON-LEIPNIK SYSTEMS BY SLIDING MODE CONTROL
}

\author{
Sundarapandian Vaidyanathan ${ }^{1}$ \\ ${ }^{1}$ Research and Development Centre, Vel Tech Dr. RR \& Dr. SR Technical University \\ Avadi, Chennai-600 062, Tamil Nadu, INDIA \\ sundarvtu@gmail.com
}

\begin{abstract}
This paper investigates the global chaos synchronization of identical hyperchaotic Newton-Leipnik systems (Ghosh and Bhattacharya, 2010) by sliding mode control. The stability results derived in this paper for the complete synchronization of identical hyperchaotic Newton-Leipnik chaotic systems are established using Lyapunov stability theory. Since the Lyapunov exponents are not required for these calculations, the sliding mode control method is very effective and convenient to achieve global chaos synchronization of the identical hyperchaotic Newton-Leipnik systems. Numerical simulations are shown to illustrate and validate the synchronization schemes derived in this paper for the identical hyperchaotic Newton-Leipnik systems.
\end{abstract}

\section{KEYWORDS}

Sliding Mode Control, Chaos Synchronization, Hyperchaos, Hyperchaotic Newton-Leipnik system.

\section{INTRODUCTION}

Chaotic systems are dynamical systems that are highly sensitive to initial conditions. The sensitive nature of chaotic systems is commonly called as the butterfly effect [1]. Synchronization of chaotic systems is a phenomenon which may occur when two or more chaotic oscillators are coupled or when a chaotic oscillator drives another chaotic oscillator. Because of the butterfly effect which causes the exponential divergence of the trajectories of two identical chaotic systems started with nearly the same initial conditions, synchronizing two chaotic systems is seemingly a very challenging problem.

In most of the chaos synchronization approaches, the master-slave or drive-response formalism is used. If a particular chaotic system is called the master or drive system and another chaotic system is called the slave or response system, then the idea of the synchronization is to use the output of the master system to control the slave system so that the output of the slave system tracks the output of the master system asymptotically.

Since the pioneering work by Pecora and Carroll ([2], 1990), chaos synchronization problem has been studied extensively and intensively in the literature [2-17]. Chaos theory has been applied to a variety of fields such as physical systems [3], chemical systems [4], ecological systems [5], secure communications [6-8], etc.

In the last two decades, various schemes have been successfully applied for chaos synchronization such as PC method [2], OGY method [9], active control method [10-12], adaptive control method [13-14], time-delay feedback method [15], backstepping design method [16], sampled-data feedback method [17], etc. 
In this paper, we derive new results based on the sliding mode control [18-20] for the global chaos synchronization of identical hyperchaotic Newton-Leipnik systems (Ghosh and Bhattacharya, [21], 2010). In robust control systems, the sliding mode control method is often adopted due to its inherent advantages of easy realization, fast response and good transient performance as well as its insensitivity to parameter uncertainties and external disturbances.

This paper has been organized as follows. In Section 2, we describe the problem statement and our methodology using sliding mode control (SMC). In Section 3, we discuss the global chaos synchronization of identical hyperchaotic Newton-Leipnik systems. In Section 4, we summarize the main results obtained in this paper.

\section{Problem Statement and Our Methodology using SMC}

In this section, we describe the problem statement for the global chaos synchronization for identical chaotic systems and our methodology using sliding mode control (SMC).

Consider the chaotic system described by

$$
\dot{x}=A x+f(x)
$$

where $x \in R^{n}$ is the state of the system, $A$ is the $n \times n$ matrix of the system parameters and $f: R^{n} \rightarrow R^{n}$ is the nonlinear part of the system.

We consider the system (1) as the master or drive system.

As the slave or response system, we consider the following chaotic system described by the dynamics

$$
\dot{y}=A y+f(y)+u
$$

where $y \in R^{n}$ is the state of the system and $u \in R^{m}$ is the controller to be designed.

If we define the synchronization error as

$$
e=y-x
$$

then the error dynamics is obtained as

$$
\dot{e}=A e+\eta(x, y)+u,
$$

where

$$
\eta(x, y)=f(y)-f(x)
$$

The objective of the global chaos synchronization problem is to find a controller $u$ such that

$$
\lim _{t \rightarrow \infty}\|e(t)\|=0 \quad \text { for all } e(0) \in R^{n} .
$$


To solve this problem, we first define the control $u$ as

$$
u=-\eta(x, y)+B v
$$

where $B$ is a constant gain vector selected such that $(A, B)$ is controllable.

Substituting (5) into (4), the error dynamics simplifies to

$$
\dot{e}=A e+B v
$$

which is a linear time-invariant control system with single input $v$.

Thus, the original global chaos synchronization problem can be replaced by an equivalent problem of stabilizing the zero solution $e=0$ of the system (7) by a suitable choice of the sliding mode control. In the sliding mode control, we define the variable

$$
s(e)=C e=c_{1} e_{1}+c_{2} e_{2}+\cdots+c_{n} e_{n}
$$

where $C=\left[\begin{array}{llll}c_{1} & c_{2} & \cdots & c_{n}\end{array}\right]$ is a constant vector to be determined.

In the sliding mode control, we constrain the motion of the system (7) to the sliding manifold defined by

$$
S=\left\{x \in R^{n} \mid s(e)=0\right\}
$$

which is required to be invariant under the flow of the error dynamics (7).

When in sliding manifold $S$, the system (7) satisfies the following conditions:

$$
s(e)=0
$$

which is the defining equation for the manifold $S$ and

$$
\dot{s}(e)=0
$$

which is the necessary condition for the state trajectory $e(t)$ of (7) to stay on the sliding manifold $S$.

Using (7) and (8), the equation (10) can be rewritten as

$$
\dot{s}(e)=C[A e+B v]=0
$$

Solving (11) for $v$, we obtain the equivalent control law

$$
v_{\text {eq }}(t)=-(C B)^{-1} C A e(t)
$$

where $C$ is chosen such that $C B \neq 0$. 
International Journal of Information Technology Convergence and Services (IJITCS) Vol.1, No.4, August 2011

Substituting (12) into the error dynamics (7), we obtain the closed-loop dynamics as

$$
\dot{e}=\left[I-B(C B)^{-1} C\right] A e
$$

The row vector $C$ is selected such that the system matrix of the controlled dynamics $\left[I-B(C B)^{-1} C\right] A$ is Hurwitz, i.e. it has all eigenvalues with negative real parts. Then the controlled system (13) is globally asymptotically stable.

To design the sliding mode controller for (7), we apply the constant plus proportional rate reaching law

$$
\dot{s}=-q \operatorname{sgn}(s)-k s
$$

where $\operatorname{sgn}(\cdot)$ denotes the sign function and the gains $q>0, k>0$ are determined such that the sliding condition is satisfied and sliding motion will occur.

From equations (11) and (14), we can obtain the control $v(t)$ as

$$
v(t)=-(C B)^{-1}[C(k I+A) e+q \operatorname{sgn}(s)]
$$

which yields

$$
v(t)= \begin{cases}-(C B)^{-1}[C(k I+A) e+q], & \text { if } s(e)>0 \\ -(C B)^{-1}[C(k I+A) e-q], & \text { if } s(e)<0\end{cases}
$$

Theorem 1. The master system (1) and the slave system (2) are globally and asymptotically synchronized for all initial conditions $x(0), y(0) \in R^{n}$ by the feedback control law

$$
u(t)=-\eta(x, y)+B v(t)
$$

where $v(t)$ is defined by $(15)$ and $B$ is a column vector such that $(A, B)$ is controllable. Also, the sliding mode gains $k, q$ are positive.

Proof. First, we note that substituting (17) and (15) into the error dynamics (4), we obtain the closed-loop error dynamics as

$$
\dot{e}=A e-B(C B)^{-1}[C(k I+A) e+q \operatorname{sgn}(s)]
$$

To prove that the error dynamics (18) is globally asymptotically stable, we consider the candidate Lyapunov function defined by the equation

$$
V(e)=\frac{1}{2} s^{2}(e)
$$

which is a positive definite function on $R^{n}$. 
Differentiating $V$ along the trajectories of (18) or the equivalent dynamics (14), we get

$$
\dot{V}(e)=s(e) \dot{s}(e)=-k s^{2}-q \operatorname{sgn}(s) s
$$

which is a negative definite function on $R^{n}$.

This calculation shows that $V$ is a globally defined, positive definite, Lyapunov function for the error dynamics (18), which has a globally defined, negative definite time derivative $\dot{V}$.

Thus, by Lyapunov stability theory [22], it is immediate that the error dynamics (18) is globally asymptotically stable for all initial conditions $e(0) \in R^{n}$.

This means that for all initial conditions $e(0) \in R^{n}$, we have $\lim _{t \rightarrow \infty}\|e(t)\|=0$.

Hence, it follows that the master system (1) and the slave system (2) are globally and asymptotically synchronized for all initial conditions $x(0), y(0) \in R^{n}$.

This completes the proof.

\section{Global Synchronization of IDENTICAl HyPERChaOtic NeWton- LEIPNIK SYSTEMS USING SLIDING MODE CONTROL}

\subsection{Theoretical Results}

In this section, we apply the sliding mode control results derived in Section 2 for the global chaos synchronization of identical hyperchaotic Newton-Leipnik systems (Ghosh and Bhattacharya, [21], 2010).

Thus, the master system is described by the hyperchaotic Newton-Leipnik dynamics

$$
\begin{aligned}
& \dot{x}_{1}=-a x_{1}+x_{2}+10 x_{2} x_{3}+x_{4} \\
& \dot{x}_{2}=-x_{1}-0.4 x_{2}+5 x_{1} x_{3} \\
& \dot{x}_{3}=b x_{3}-5 x_{1} x_{2} \\
& \dot{x}_{4}=-c x_{1} x_{3}+d x_{4}
\end{aligned}
$$

where $x_{1}, x_{2}, x_{3}, x_{4}$ are state variables and $a, b, c, d$ are positive, constant parameters of the system.

The slave system is also described by the controlled hyperchaotic Newton-Leipnik dynamics

$$
\begin{aligned}
& \dot{y}_{1}=-a y_{1}+y_{2}+10 y_{2} y_{3}+y_{4}+u_{1} \\
& \dot{y}_{2}=-y_{1}-0.4 y_{2}+5 y_{1} y_{3}+u_{2} \\
& \dot{y}_{3}=b y_{3}-5 y_{1} y_{2}+u_{3} \\
& \dot{y}_{4}=-c y_{1} y_{3}+d y_{4}+u_{4}
\end{aligned}
$$

where $y_{1}, y_{2}, y_{3}$ are state variables and $u_{1}, u_{2}, u_{3}$ are the controllers to be designed. 
International Journal of Information Technology Convergence and Services (IJITCS) Vol.1, No.4, August 2011

The systems (21) and (22) are hyperchaotic when

$$
a=0.4, b=0.175, c=0.8 \text { and } d=0.01 .
$$

Figure 1 illustrates the state portrait of the hyperchaotic Newton-Leipnik system (21).
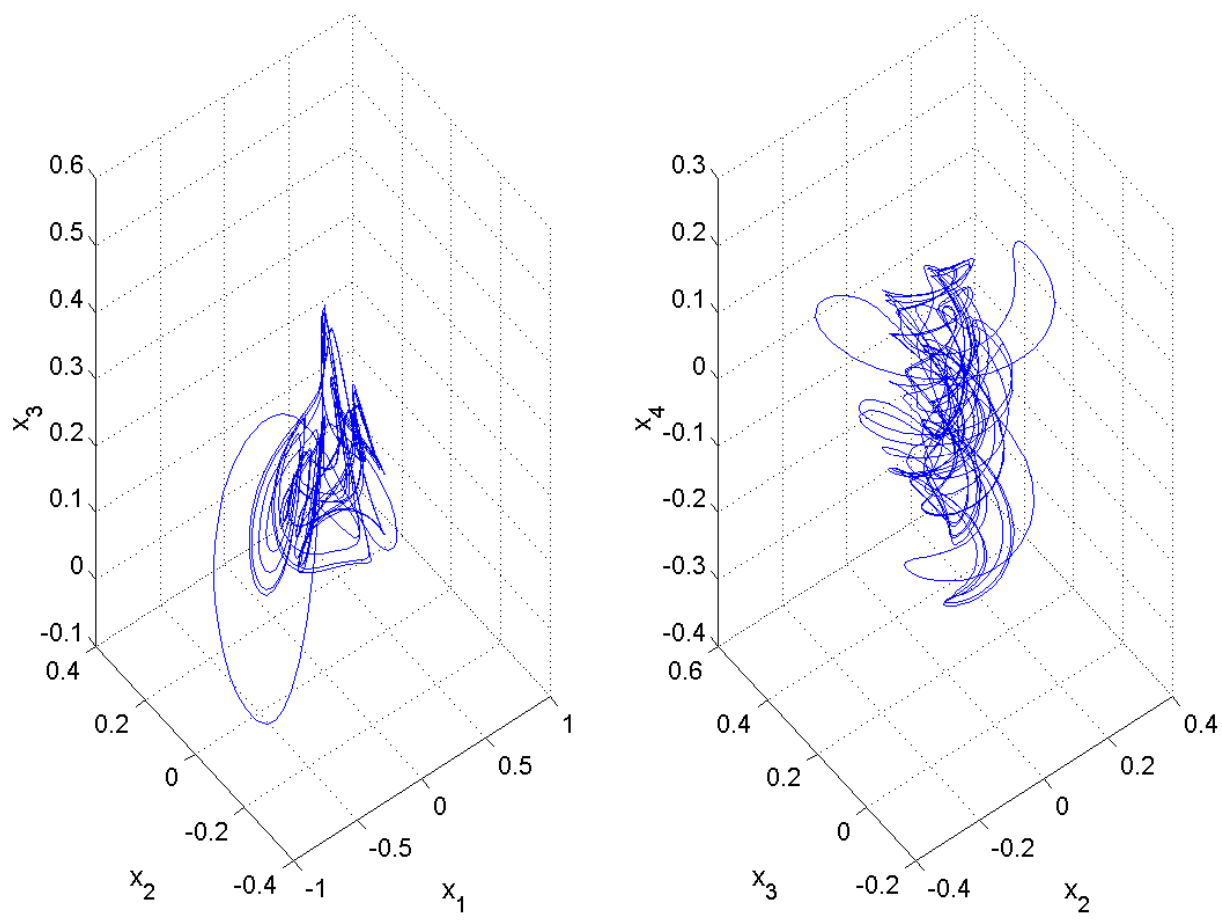

Figure 1. State Orbits of the Hyperchaotic Newton-Leipnik System

The chaos synchronization error is defined by

$$
e_{i}=y_{i}-x_{i},(i=1,2,3)
$$

The error dynamics is easily obtained as

$$
\begin{aligned}
& \dot{e}_{1}=-a e_{1}+e_{2}+e_{4}+10\left(y_{2} y_{3}-x_{2} x_{3}\right)+u_{1} \\
& \dot{e}_{2}=-e_{1}-0.4 e_{2}+5\left(y_{1} y_{3}-x_{1} x_{3}\right)+u_{2} \\
& \dot{e}_{3}=b e_{3}-5\left(y_{1} y_{2}-x_{1} x_{2}\right)+u_{3} \\
& \dot{e}_{4}=-c\left(y_{1} y_{3}-x_{1} x_{3}\right)+d e_{4}+u_{4}
\end{aligned}
$$

We write the error dynamics (24) in the matrix notation as

$$
\dot{e}=A e+\eta(x, y)+u
$$

where 


$$
A=\left[\begin{array}{cccc}
-a & 1 & 0 & 1 \\
-1 & -0.4 & 0 & 0 \\
0 & 0 & b & 0 \\
0 & 0 & 0 & d
\end{array}\right], \eta(x, y)=\left[\begin{array}{c}
10\left(y_{2} y_{3}-x_{2} x_{3}\right) \\
5\left(y_{1} y_{3}-x_{1} x_{3}\right) \\
-5\left(y_{1} y_{2}-x_{1} x_{2}\right) \\
-c\left(y_{1} y_{3}-x_{1} x_{3}\right)
\end{array}\right] \text { and } u=\left[\begin{array}{c}
u_{1} \\
u_{2} \\
u_{3} \\
u_{4}
\end{array}\right]
$$

The sliding mode controller design is carried out as detailed in Section 2.

First, we set $u$ as

$$
u=-\eta(x, y)+B v
$$

where $B$ is chosen such that $(A, B)$ is controllable.

We take $B$ as

$$
B=\left[\begin{array}{l}
1 \\
1 \\
1 \\
1
\end{array}\right] .
$$

In the hyperchaotic case, the parameter values are

$$
a=0.4, b=0.175, c=0.8 \text { and } d=0.01 \text {. }
$$

The sliding mode variable is selected as

$$
s=C e=\left[\begin{array}{llll}
0.4 & 4 & 8 & 0.1
\end{array}\right] e=0.4 e_{1}+4 e_{2}+8 e_{3}+0.1 e_{4}
$$

which makes the sliding mode state equation asymptotically stable.

We choose the sliding mode gains as $k=5$ and $q=0.1$.

We note that a large value of $k$ can cause chattering and an appropriate value of $q$ is chosen to speed up the time taken to reach the sliding manifold as well as to reduce the system chattering.

From Eq. (15), we can obtain $v(t)$ as

$$
v(t)=0.1728 e_{1}-1.5040 e_{2}-3.3120 e_{3}-0.0721 e_{4}-0.0080 \operatorname{sgn}(s)
$$

Thus, the required sliding mode controller is obtained as

$$
u=-\eta(x, y)+B v
$$

where $\eta(x, y), B$ and $v(t)$ are defined as in the equations (26), (28) and (30). 
By Theorem 1, we obtain the following result.

Theorem 2. The identical hyperchaotic Newton-Leipnik systems (21) and (22) are globally and asymptotically synchronized for all initial conditions with the sliding mode controller udefined by (31).

\subsection{Numerical Results}

In this section For the numerical simulations, the fourth-order Runge-Kutta method with timestep $h=10^{-6}$ is used to solve the hyperchaotic Newton-Leipnik systems (21) and (22) with the sliding mode controller $u$ given by (31) using MATLAB.

The sliding mode gains are chosen as $\quad k=5$ and $q=0.1$.

The initial values of the master system $(21)$ are taken as $x(0)=(14,5,8,6)$ and the initial values of the slave system (22) are taken as $(9,2,12,1)$.

Figure 2 illustrates the complete synchronization of the identical hyperchaotic Newton-Leipnik systems (21) and (22).

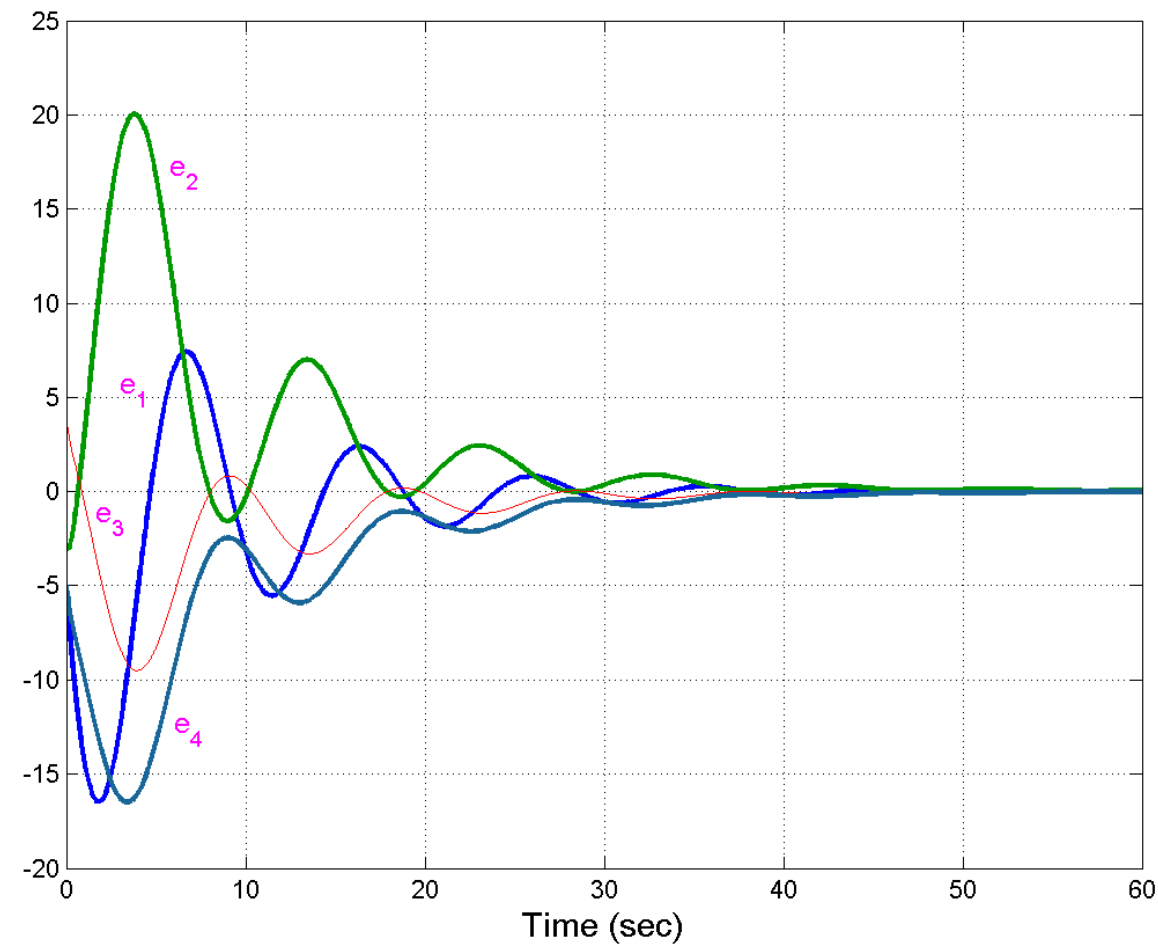

Figure 2. Synchronization of Identical Hyperchaotic Newton-Leipnik Systems 


\section{Conclusions}

In this paper, we have deployed sliding mode control (SMC) to achieve global chaos synchronization for the identical hyperchaotic Newton-Leipnik systems (2010). Our synchronization results for the identical hyperchaotic Newton-Leipnik systems have been proved using Lyapunov stability theory. Since the Lyapunov exponents are not required for these calculations, the sliding mode control method is very effective and convenient to achieve global chaos synchronization for the identical hyperchaotic Newton-Leipnik systems. Numerical simulations are also shown to illustrate the effectiveness of the synchronization results derived in this paper using the sliding mode control.

\section{REFERENCES}

[1] Alligood, K.T., Sauer, T. \& Yorke, J.A. (1997) Chaos: An Introduction to Dynamical Systems, Springer, New York.

[2] Pecora, L.M. \& Carroll, T.L. (1990) "Synchronization in chaotic systems", Phys. Rev. Lett., Vol. 64, pp 821-824.

[3] Lakshmanan, M. \& Murali, K. (1996) Nonlinear Oscillators: Controlling and Synchronization, World Scientific, Singapore.

[4] Han, S.K., Kerrer, C. \& Kuramoto, Y. (1995) "Dephasing and burstling in coupled neural oscillators", Phys. Rev. Lett., Vol. 75, pp 3190-3193.

[5] Blasius, B., Huppert, A. \& Stone, L. (1999) "Complex dynamics and phase synchronization in spatially extended ecological system”, Nature, Vol. 399, pp 354-359.

[6] Cuomo, K.M. \& Oppenheim, A.V. (1993) "Circuit implementation of synchronized chaos with applications to communications," Physical Review Letters, Vol. 71, pp 65-68.

[7] Kocarev, L. \& Parlitz, U. (1995) "General approach for chaotic synchronization with applications to communication," Physical Review Letters, Vol. 74, pp 5028-5030.

[8] Tao, Y. (1999) "Chaotic secure communication systems - history and new results," Telecommun. Review, Vol. 9, pp 597-634.

[9] Ott, E., Grebogi, C. \& Yorke, J.A. (1990) "Controlling chaos”, Phys. Rev. Lett., Vol. 64, pp 1196-1199.

[10] Ho, M.C. \& Hung, Y.C. (2002) "Synchronization of two different chaotic systems using generalized active network," Physics Letters A, Vol. 301, pp 424-428.

[11] Huang, L., Feng, R. \& Wang, M. (2005) "Synchronization of chaotic systems via nonlinear control," Physical Letters A, Vol. 320, pp 271-275.

[12] Chen, H.K. (2005) "Global chaos synchronization of new chaotic systems via nonlinear control," Chaos, Solitons \& Fractals, Vol. 23, pp 1245-1251.

[13] Lu, J., Wu, X., Han, X. \& Lü, J. (2004) “Adaptive feedback synchronization of a unified chaotic system,” Physics Letters A, Vol. 329, pp 327-333.

[14] Chen, S.H. \& Lü, J. (2002) "Synchronization of an uncertain unified system via adaptive control," Chaos, Solitons \& Fractals, Vol. 14, pp 643-647.

[15] Park, J.H. \& Kwon, O.M. (2003) "A novel criterion for delayed feedback control of time-delay chaotic systems," Chaos, Solitons \& Fractals, Vol. 17, pp 709-716.

[16] Wu, X. \& Lü, J. (2003) "Parameter identification and backstepping control of uncertain Lü system," Chaos, Solitons \& Fractals, Vol. 18, pp 721-729.

[17] Zhao, J. \& J. Lu (2006) "Using sampled-data feedback control and linear feedback synchronization in a new hyperchaotic system," Chaos, Solitons \& Fractals, Vol. 35, pp 376382. 
[18] Slotine, J.E. \& Sastry, S.S. (1983) "Tracking control of nonlinear systems using sliding surface with application to robotic manipulators," Internat. J. Control, Vol. 38, pp 465-492.

[19] Utkin, V.I. (1993) "Sliding mode control design principles and applications to electric drives," IEEE Trans. Industrial Electronics, Vol. 40, pp 23-36, 1993.

[20] Saravanakumar, R., Vinoth Kumar, K. \& Ray, K.K. (2009) "Sliding mode control of induction motor using simulation approach," Internat. J. Control of Comp. Sci. Network Security, Vol. 9, pp 93-104.

[21] Ghosh, D. \& Bhattacharya, S. (2010) "Projective synchronization of new hyperchaotic system with fully unknown parameters," Nonlinear Dynamics, Vol. 61, pp 11-21.

[22] Hahn, W. (1967) The Stability of Motion, Springer, New York.

\section{Author}

Dr. V. Sundarapandian is a Professor (Systems and Control Engineering), Research and Development Centre at Vel Tech Dr. RR \& Dr. SR Technical University, Chennai, India. His current research areas are: Linear and Nonlinear Control Systems, Chaos Theory, Dynamical Systems and Stability Theory, Soft Computing, Operations Research, Numerical Analysis and Scientific Computing, Population Biology, etc. $\mathrm{He}$ has published over 170 research articles in international journals and two text-books with Prentice-Hall of India, New Delhi, India. He has published over 50 papers in International Conferences and 90 papers in National Conferences. He is the Editor-in-Chief of the AIRCC Journals - International Journal of Instrumentation and Control Systems, International Journal of Control Theory and Computer Modeling, etc. $\mathrm{He}$ is an Associate Editor of International Journal of Control Theory and Applications, International Journal of Computer Information Systems, International Journal of Advances in Science and Technology, etc. He has delivered several Key Note Lectures on Control Systems, Chaos Theory, Scientific Computing, MATLAB, SCILAB, etc. 